\title{
Los territorios de la Submeseta sur en época de Augusto*
}

\author{
Gregorio Carrasco Serrano \\ Universidad de Castilla-La Mancha \\ gregorio.carrasco@uclm.es
}

\section{RESUMEN}

El presente trabajo se centra en los ámbitos más meridionales de la Meseta en época augustea, teniéndose en cuenta, por tanto, aquellos principales núcleos de población, con sus respectivos territorios, que recibieron el impulso del programa de actuación de Augusto en estas tierras, llegando a alcanzar estatuto jurídico privilegiado en dicha época. Este será el caso de Oretum sobre el valle del río Jabalón, o de la colonia Libisosa de sobrenombre Foroaugustana, en la parte occidental de la provincia de Albacete. Estos núcleos de población, situados junto a los principales ejes de comunicación, van a constituirse en centros fundamentales de la ordenación y organización territorial en estos ámbitos de la Submeseta sur.

Palabras clave: Meseta meridional. Territorios. Núcleos de población. Ejes de comunicación.

\section{The Territories of the Southern Meseta in the Augustan Era}

\begin{abstract}
This work focuses on the southernmost area of the Meseta in the Augustan era, thus taking into account those main settlements and the respective territories thereof that received the boost of the action program of Augustus in these lands thereby reaching a privileged legal statute at that moment. This is the case of Oretum over the valley of the Jabalón river, or the colony of Libisosa having the surname Foroaugustana in the western part of the province of Albacete. These settlements located next to the main transport axes, will become fundamental centers of the territorial planning and organization in these areas of the southern part of the Meseta.
\end{abstract}

Key Words: Southern Meseta. Territories. Settlements. Communications axes. 
Los territorios meridionales de la Meseta quedarían bajo total control romano a partir ya de mediados del siglo II a.C. ${ }^{1} \mathrm{Y}$ en su mayor parte, dichos ámbitos territoriales se integrarían en el nuevo sistema organizativo romano adscribiéndose a la Citerior. Posteriormente la importante reorganización territorial llevada a cabo por Augusto, afectaría obviamente a estas tierras de la Meseta sur que pasarían a integrarse en su mayoría, desde un punto de vista político-administrativo, en la Tarraconense, ${ }^{2}$ a excepción de determinados ámbitos; este será el caso por ejemplo del territorio del suroeste de la actual provincia de Ciudad Real que quedaría adscrito en un principio a la Bética. Por otro lado, la mayor parte de estas tierras más meridionales de la Meseta sur, se enmarcarán dentro del conventus Carthaginiensis, excepto la parte suroccidental de la provincia de Ciudad Real que -y tal como se ha indicado-, perteneciendo a la provincia Bética, quedaría adscrita inicialmente al conventus Cordubensis.

Pero en estos territorios de la Submeseta sur se van a articular diversos ejes de comunicación, que van a constituir elementos fundamentales en la vertebración y en la ordenación del poblamiento y del propio territorio. Entre estos ejes de comunicación destaca en primer término el denominado Per Lusitaniam ab Emerita Caesarea Augusta ${ }^{3}$ recogido por el Itinerario de Antonino (It. Ant. 444.3 ss). Se trata de un eje que desde la capital de la Lusitania, Emerita, se extendía hasta Caesaraugusta a través de una serie de tramos claramente diferenciados. ${ }^{4}$ Pues bien, este eje atravesaría longitudinalmente de oeste a este, y en uno de dichos tramos, el ámbito territorial correspondiente a la provincia de Ciudad Real, se concentró toda una serie de núcleos de población algunos de los cuales llegarían a alcanzar estatuto jurídico privilegiado, incluso probablemente según G. Alföldy desde comienzos de época imperial.

El primero de estos núcleos que cabe mencionar es Sisapo, ${ }^{5}$ ubicado en el suroeste de la provincia de Ciudad Real, en el valle de Alcudia. Son diversas las fuentes antiguas que hacen referencia a Sisapo en relación sobre todo a su importancia minera. Así pues, ya se cita en forma explícita por Estrabón (III 2.3), quien alaba su riqueza metalífera, diferenciando además un núcleo antiguo y otro nuevo. ${ }^{6}$ Pero será sin duda el naturalista Plinio, quien aporte más datos sobre la explotación del mineral sisaponense. Así pues, según dicho autor clásico (XXXIII 118) el minio de Sisapo era el más conocido, ${ }^{7}$ siendo transportado a Roma en bruto y bajo precinto; añade además

\footnotetext{
* Dentro de la Meseta sur el presente trabajo se centra en los territorios correspondientes a la provincia de Ciudad Real y parte occidental del ámbito provincial de Albacete.

1 Carrasco Serrano 2008, 30.

2 Sobre la Citerior Tarraconense en época alto-imperial, véase la reciente obra de OzCÁRIz GIL 2013.

3 Este eje viario es denominado por Saavedra $(1862,76)$ con el número 29 Per Lusitaniam ab Emerita Caesaraugustam.

4 Vid. RoldÁn Hervás 1975, 91-93.

5 En relación al nombre vid. Holder 1904, 1584; Villar 2000, 170, 342, 383 y 440; García Alonso $2003,344$.

6 Según García y Bellido 1945, 73, Estrabón debería referirse a la ciudad ibérica y a la romana. Véase también al respecto Schulten 1952, 159; AbASCAL-ESPinosa 1989, 28-29; Bendala Galán 1990, 36, n.53; Abad Casal - Bendala Galán 1997, 17; Bendala Galán 1998, 309. En cuanto a la diferente localización de la Sisapo vetus y nova, vid. Stylow 2000, 791; también Canto 1993, 183.

7 En consonancia con Plinio, Trogo Pompeyo afirma también que Hispania era la región que más minio producía (Iust., Epit. Hist. Ph. XLIV 1.6: Sed nec summae tantum terrae laudanda bona, verum et abstru-
} 
Plinio que con frecuencia se adulteraba de distintas formas, lo cual proporcionaba grandes beneficios a las compañías que lo trabajaban. ${ }^{8}$

También Vitruvio, ${ }^{9}$ contemporáneo de Augusto, atestigua de forma explícita la preparación del minio en talleres de Roma. En época de Cicerón (Ph. II 48), la explotación de estas minas estaría en manos de una compañía o societas, ${ }^{10}$ como se constata epigráficamente en CIL VI, 9634 y CIL X, 3964. A estos últimos testimonios hay que añadir también un muy interesante epígrafe procedente de las inmediaciones de Córdoba ( $C I L I I^{2} / 7,699$ a), en el que se hace mención a la servidumbre de paso impuesta por la societas sisaponensis ${ }^{11}$ en relación a la vía que enlazaba Sisapo con Corduba, ${ }^{12}$ ruta esta que permitiría hacer llegar hasta esta última ciudad, de privilegiada situación estratégica dentro del gran eje del Guadalquivir, los productos mineros de la comarca sisaponense, y muy especialmente el mercurio utilizado en las técnicas de amalgamación de metales nobles. ${ }^{13}$

Asimismo carácter minero tendrá la vía que unía Sisapo con Castulo; una ruta esta atestiguada a través de la inscripción $C I L$ II, $3270,{ }^{14}$ en la que se constata la labor benefactora realizada por $Q$. Torius Culleo ${ }^{15}$ procurator Augusti provinciae Baeticae, quien llevaría a cabo la reparación de dicha vía ${ }^{16}$ que a través del Salto Castulonense conducía a Sisapo (... viam quae per Castul(onensem) saltum Sisaponem ducit). A través de esta ruta quedarían, pues, unidos estos dos grandes centros de distritos mi-

sorum metallorum felices divitiae, iam lini spartique vis ingens, minii certe nulla feracior terra). Plinio en relación al minio sisaponense añade en XXXIII, 121: Sisaponensibus autem miniariis sua vena harena sine argento. Excoquitur auri modo. Probatura uro candente, fucatum enim nigrescit, sincerum retinet colorem. Por otra parte SCHULTEN $(1952,159)$, pone en duda que la cita de Teofrasto sobre el cinabrio se refiera al sisaponense; vid. también GARCía y BELLIDO 1953, 440.

8 Plin. XXXIII 118: Iuba minium nasci et in Carmania tradit; Timagenes et in Aethiopia, sed neutro ex loco invehitur ad nos nec fere aliunde quam ex Hispania, celeberrimo Sisaponensi regione in Baetica miniario metallo vectigalibus populi Romani nullius rei deligentiore custodia. Non licet ibi perficere id excoquique: Romam adfertum vena signata ad bina milia fere pondo annua; Romae autem lavatur in vendendo pretio statuta lege, ne modum excederet HS LXX in libras; sed adulteratur multis modis, unde praeda societati.

9 Vitr., VII 9.4: quae autem in Ephesiorum metallis fuerunt officinae, nunc traeiectae sunt ideo Romam, quod id genus venae postea inventum Hispaniae regionibus, e quibus metallis glaebae portantur et per publicanus Romae curantor. Eae autem officinae sunt inter eadem Florae et Quirini.

10 Vid. Thouvenot 1940, 185-186 y 262; Domergue 1990, 193, 214, 269-270, 379; ID. 2008, 70.

11 Cf. Ventura Villanueva 1993, 49-61. Véase también al respecto la muy interesante inscripción funeraria procedente igualmente de Córdoba, en la que se constata a tres libertos de la Societas Sisaponensis, y que portan curiosamente el nomen de Argentarius: M(arcus). Argentarius. s(ocietatis). S(isaponensis). l(ibertus). Philinus / A(ulus). Argentarius. s(ocietatis). S(isaponensis). l(ibertus). Rufus / M(arcus). Argentarius. s(ocietatis). S(isaponensis). l(ibertus) / Succio / suo. testamento fieri / iussit. Vid. CIL II $\mathrm{I}^{2} 7415 \mathrm{a}$.

12 Sillières (1990, 416-500), ha propuesto un posible trazado para esta vía, basándose en la ruta de AlIdrisi, Toledo-Córdoba. No obstante véase también al respecto CoRCHADo SoriAno 1969a; ID. 1969b, $137-$ 138; también y más recientemente MelCHOR GIL 1993, 69-71; ID. 1995, 151-153.

13 Vid. CHIC García 1991, 7-29.

14 Véase también Vives 1971, 167-168, nº1417; GonzÁlez Román - MANGas 1991, 144-145, n91. En general y sobre dicha vía, vid. CARRASCO SERRANO 1997, 183-191.

15 Vid. Contreras de la Paz 1965, 63-96; DunCan-Jones 1974, 19-85.

16 Cf. Sillières 1990, 490-493; Corzo Sánchez - Toscano San Gil 1992, 53, 185 y 224. 
neros, como serían Sisapo en el suroeste de la provincia de Ciudad Real y Castulo en el norte de la provincia de Jaén. ${ }^{17}$

Por otra parte Sisapo es mencionado por Plinio (III 14) entre los núcleos de la Beturia túrdula, sin embargo en Ptolomeo (II 6.58) se incluye en la Citerior, lo cual podría explicarse, según Thouvenot, ${ }^{18}$ por la rectificación de límites provinciales que debió efectuarse ya avanzado el siglo I d.C. De esta manera el territorio de Sisapo pasaría a formar parte de la provincia imperial Citerior Tarraconense, de cara probablemente a garantizar un mejor control sobre este tan destacable distrito minero.

Situado, como ya se ha indicado (vid. supra), en el eje de comunicación ${ }^{19}$ recogido por el Itinerario de Antonino Per Lusitaniam ab Emerita Caesarea Augusta, a XIII m.p. de Mirobriga y XX m.p. de Carcuvium, su localización exacta ha venido planteando toda una serie de problemas. Así pues, ha sido frecuentemente identificada con Almadén, por Madoz, ${ }^{20}$ Saavedra, ${ }^{21}$ Coello, ${ }^{22}$ Blázquez, ${ }^{23}$ Miller ${ }^{24}$ y posteriormente ya por Schulten ${ }^{25}$ y García Bellido ${ }^{26}$ entre otros. ${ }^{27}$ Por su parte Flórez ${ }^{28}$ la colocaría en Valdeazogues, mientras que Corchado Soriano ${ }^{29}$ siguiendo a Hervás y Buendía ${ }^{30}$ se inclinaría por Chillón. ${ }^{31}$ Más recientemente Sillières ${ }^{32}$ propuso su localización en el Cerro de las Monas, sin embargo los recientes hallazgos arqueológicos y sobre todo epigráficos ${ }^{33}$ realizados en La Bienvenida (término de Almodóvar del Campo) han venido a reforzar últimamente su identificación en dicho emplazamiento. ${ }^{34}$ Unos hallazgos epigráficos que por otra parte han permitido confirmar el estatuto jurídico municipal alcanzado por este núcleo de población.

17 Hay que tener en cuenta la opinión expresada por DunCAN-Jones 1974, 80, según la cual no pueden ser excluidas posibles ventajas que para la explotación de las minas de plata de Cástulo, tendría el comercio con Sisapo.

18 Thouvenot 1940, 164-165 y 248. Véase también sobre dicha cuestión Albertini 1923, 114-115; GARCía Iglesias 1971, 102-103; Prieto Arciniega 1973, 134; Marín Díaz - Prieto Arciniega 1974, 82; RolDÁN Hervás 1982, 100; Plácido 2008, 37.

19 En relación a la viaria en torno a Sisapo, vid. CArrasco SErrano 2007, 363-74.

20 MAdOZ 1846, 21.

21 SAAVEDRA $1862,103$.

22 Coello 1889, 20.

23 BlázQuez 1892, 124; ID. 1912, 368.

24 Miller 1916, 181.

25 SChulten 1952, 159.

26 GARCía y BELLIDO 1945, 73; ID. 1947, 228 y 277.

27 También realizan dicha identificación de Sisapo en Almadén, AlberTini 192, 90 y 114; ThOUVENOT 1940, 165 y 279; García IgLesias 1971, 103.

28 FLÓREZ 1751, VII, 140.

29 CORChado Soriano 1969b, 156; ID. 1982, 43.

30 HeRvás y BuENdía 1899, 304.

31 Véase también al respecto FiTA 1910, 527-528.

32 SiLliÈres 1980, 49-57.

33 Vid. CIL II $2 / 7,179-180$. También al respecto Fernández OCHOA ET ALII 1982-83, 211-220; ALFÖLDY 1987, 56-57; Gimeno Pascual 2008, 324-325.

34 Vgr. véase Stylow 1985, 658; Alföldy 1987, 55. De todos modos, sobre la falta de equivalencia en las distancias entre mansiones que el Itinerario de Antonino ofrece por lo que respecta a Sisapo-La Bienvenida y Carcuvium, vid. SiLlières 1990, 374-375 y 378-379. 
Ya Alföldy ${ }^{35}$ plantearía el carácter de comunidad privilegiada para Sisapo en base, entre otros criterios, a la epigrafía, proponiendo su promoción jurídica a comienzos de época imperial. ${ }^{36}$ Dicha promoción jurídica, que quizá podría haber sido paralela a su auge y desarrollo urbano experimentados en tiempos augusteos, ${ }^{37}$ se vería no cabe duda posibilitada por su gran importancia como centro minero. Por otro lado el territorio de influencia de Sisapo debió de ser amplio, teniendo en cuenta dicha destacable función como centro minero, extendiéndose por toda la zona del valle de Alcudia, e integrando a toda una serie de poblados y núcleos mineros metalúrgicos ${ }^{38}$ dentro de una ordenación territorial perfectamente organizada.

Junto a Sisapo, otro de los núcleos de población en este ámbito territorial de la submeseta sur que llegaría a alcanzar estatuto jurídico privilegiado de carácter municipal, incluso ya en época augustea, sería Oretum..$^{39}$ Situado en el valle del Jabalón, es mencionado por Artemidoro de Éfeso a través de Esteban de Bizancio con el nombre de ' $\Omega$ pıбía, mientras que en Estrabón (III 3.2) se cita con la forma de ' $\Omega$ pía, como núcleo también más importante junto con Cástulo de la región oretana. ${ }^{40}$ Por su parte Ptolomeo (II 6.58) la recoge igualmente en su relación de ciudades de la Oretania con

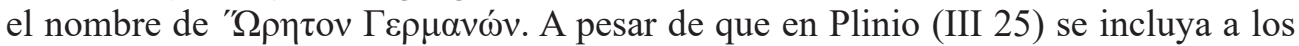
Oretani qui et Germani cognominantur, entre las principales ciudades estipendiarias (stipendiariorum autem celeberrime...) del conventus Carthaginiensis, ${ }^{41}$ sin embargo del testimonio epigráfico puede deducirse que alcanzaría status jurídico privilegiado. ${ }^{42}$ Así pues, de dicho núcleo procede la inscripción CIL II, $3221,{ }^{43}$ conservada en la actualidad en el ayuntamiento de Almagro, en la que se constata la labor evergética llevada a cabo en la ciudad ${ }^{44}$ por parte de P(ublius) Baebius Venustus, un posible miembro de la élite local. Ya Alföldy ${ }^{45}$ propondría una vinculación saguntina para Baebius Venustus, poniendo de manifiesto la presencia de ciertos grupos familiares procedentes del Levante en estas ciudades de la Meseta sur, en donde van a adquirir una posición de relevancia social.

35 AlFÖLDY 1987, 56-57.

36 Alföldy 1987, 56; ID. 1999, 473; AbascAl - Espinosa 1989, 66; Andreu Pintado 2008, $236-237$. No obstante téngase en cuenta también las reservas planteadas por STYLOW (1991, 11-27), en relación a la municipalización preflavia de Sisapo.

37 Vid. Zarzalejos Prieto ET ALII 1994, 184-185; FernÁNDEZ OCHOA ET ALII 1994, 154-155; ZarZALEJOS ET ALII 2004, 172-173; FERNÁNDEZ OCHOA ET ALII 2002, 55.

38 FERNÁNDEZ OCHOA ET ALII 2002.

39 En relación al nombre vid. Tovar 1980, 181; VILlar 2000, 135, 194, 199-200, 202, 205, 284, 358,393

y 429; García Alonso 2003, 345-346; ID. 2008, 354.

40 SCHUlten 1952, 200-201.

41 Plin., III 25: stipendiariorum autem celeberrimi Alabanenses, Bastitani, Consaburrenses, Dianenses, Egelestani, Ilorcitani, Laminitani, Mentesani qui et Oretani, Mentesani qui et Bastuli, Oretani qui et Germani cognominantur (...).

42 Vid. Alföldy 1987, 46-52; Abascal-Espinosa 1989, 66; Carrasco Serrano 1999, 321-322.

43 Vives 1971, 244, n'2067.

44 Melchor Gil 1999, 237 y 242. También véase Piernavieja 1977, 124; Humphrey 1986, 360; Sillières 1990, 165-166. Más recientemente, Gimeno Pascual 2008, 325; Velaza 2012, 399.

45 Alföldy 1987, 48-49; AbasCAL 2004, 148. 
Según Alföldy la promoción jurídica de Oretum se llevaría a cabo, muy posiblemente, en tiempos de Augusto. ${ }^{46}$ Por otra parte su localización se viene tradicionalmente situando en torno a la ermita de Nuestra Sra. de Zuqueca o de Oreto, ${ }^{47}$ que conserva el antiguo nombre, en el término municipal de Granátula de Calatrava, en una posición privilegiada de cara a las comunicaciones. ${ }^{48}$ El territorio de Oretum se extendería por buena parte de la actual comarca del Campo de Calatrava, incluyendo probablemente a Carcuvium ${ }^{49}$ que aparece citada como mansio (It. Ant. 445.1), como ya se ha indicado, en este eje correspondiente a la vía n ${ }^{\circ} 29$ del Itinerario de Antonino. Y precisamente de Carcuvium procede una inscripción ${ }^{50}$ en la que se documenta la tribu Galeria, a la que pertenecía tan solo uno de los dos magistrados atestiguados en dicho epígrafe. Por tanto Carcuvium, según Alföldy ${ }^{51}$ se trataría más bien de un vicus dependiente en el territorio de una ciudad próxima que podría ser Oretum.

También en este eje de comunicación (Per Lusitaniam ab Emerita Caesarea Augusta) recogido por el Itinerario de Antonino, como elemento vertebrador del poblamiento en estos territorios hay que ubicar a Laminium. Un núcleo este que -siendo municipio flavio- es probable se viese ya impulsado desde comienzos del siglo I d.C., según se deduce del testimonio epigráfico (vid. infra). Laminium ${ }^{52}$ vendrá a constituir un destacable centro de intersección viario en la estructura de las comunicaciones de estas tierras de la Submeseta sur. Así pues es mencionada por el Itinerario de Antonino en tres de los principales ejes viarios de este ámbito territorial. En primer término en el ya citado Per Lusitaniam ab Emerita Caesarea Augusta, situado como mansio (It. Ant. 445.4) entre Mariana y Alces. También será punto de arranque del eje que conducía a Toletum, Item a Laminio Toletum (It. Ant. 446.4-7), e igualmente en la segunda ruta dada por el Itinerario de Antonino para comunicar Laminio con Caesaraugusta, Item a Laminio alio itinere Caesarea Augusta (It. Ant. 446.8-448.1) frente a 445, 4-446, 3. En este último caso, desde Laminium, pues dicho eje continuaría por el nacimiento del Guadiana (Caput fluminis Anae), ${ }^{53}$ en dirección a la colonia de Libisosa (Lezuza) para proseguir posteriormente a través de otra serie de mansiones

\footnotetext{
46 Alföldy 1987, 52; ID. 1987a, 237; Olivares Pedreño 1998, 222; AlföLdy 1999, 473; AbasCAL 2006, 77.

47 Vid. SaAvedra 1862, 99; Hervás y Buendía 1899, 358; Miller 1916, 159; SCHulten 1952, 201; GarCía y Bellido 1947, 238; Corchado Soriano 1969b, 154; ID. 1982, 260 ss; Alföldy 1987, 47; Sillières 1990, 337. En relación a las excavaciones llevadas a cabo en el Cerro Domínguez y Oreto-Zuqueca, véase Nieto Gallo Et ALII 1980; Garcés ET ALII 2000, 241-255; GarcÉs-Romano 2004, 307-324.

48 Corchado Soriano 1969. También y según Sillières (1990, 493-496), Oretum estaría comunicado con Castulo mediante una ruta a través de Sierra Morena. Por otra parte y aunque no sea citada por ninguna fuente itineraria antigua, es muy posible que el eje de comunicación recogido por el Itinerario de Antonino (Per Lusitaniam ab Emerita Caesarea Augusta) pasase por Oretum, vid. Alföldy 1987, 47; SilliÉRES 1990, 377.

49 Sobre el nombre vid. Villar 2000, 160, 165, 175, 283, 290, 306, 383-4 y 393; GARCía Alonso 2008, 348.

50 ALFÖLDY 1987a, 236-237.

51 ALFÖLDY 1987, 50-51; ID. 1987a, 237; ID. 1999, 474. No obstante más recientemente véase al respecto Ortiz de Urbina Álava 2000, 136, nota 292.

52 En cuanto al nombre vid. Holder 1904, 130; Curchin 1997, 268; García Alonso 2003, 325-326; ID. $2008,351$.

53 Sobre la problemática de su identificación vid. CARrasCo SERrano 1990, 91; ID. 2011, 329.
} 
hacia Caesaraugusta. También el Anónimo de Rávena (313.17) lo incluye en uno de sus trazados descritos. ${ }^{54}$ Por tanto este muy favorable emplazamiento en el entramado viario de la zona, ${ }^{55}$ permitiría sin duda a Laminium mantener relaciones con otros ámbitos peninsulares sobre todo del Levante y Mediodía. Por su parte Plinio (III 6) pone precisamente el origen del río Anas en el ager Laminitanus,${ }^{56}$ afirmando además y por otro lado (XXXVI 165) que las mejores piedras de afilar instrumentos de hierro procedían de Laminium; ${ }^{57}$ referencia esta última atestiguada claramente a través de la existencia de importantes canteras en los alrededores de la localidad de Alhambra ${ }^{58}$ (en donde últimamente se viene situando dicho enclave), y cuya explotación muy posiblemente constituyera un elemento clave en la economía de la comarca en época romana.

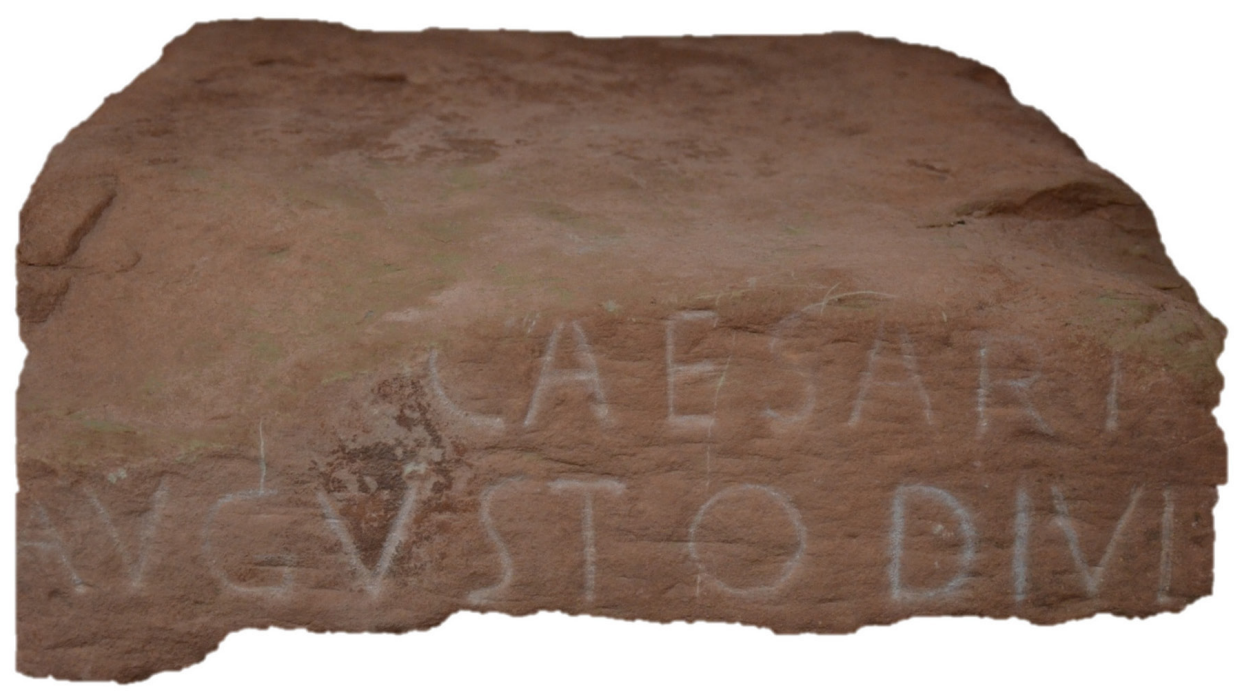

Fig. 1. Pedestal en honor a Augusto procedente de Alhambra (Ciudad Real).

Aun cuando Plinio (III 25) incluya a los Laminitani entre las comunidades estipendiarias del conventus Carthaginiensis, sin embargo epigráficamente ${ }^{59}$ puede cons-

54 Roldán Hervás 1975, 129.

55 Precisamente de Alhambra (Laminium) procede un epígrafe votivo dedicado muy significativamente a Mercurio, deidad protectora de caminos, encrucijadas y del comercio, vid. Alföldy 1987a, 246-247; CORELL 1996, n²34; BARATTA 2001, 24-25.

56 Plin., III 6: Vlterior in duas per longitudinemn provincias dividitur, siquidem Baeticae latere septentrionali praetenditur Lusitania amne Ana discreta. Ortus hic in Laminitano agro Citerioris Hispaniae et modo in stagna se fundens, modo in angustias resorbens aut in totum cuniculis condens et saepius nasci gaudens in Atlanticum Oceanum effunditur. Vid. Schulten 1963 II, 66; García y Bellido 1947, 210.

57 Plin., XXXVI 165: Quarta ratio est saliva hominis cotium proficientium in tonstrinarum officinis: Laminitanae ex Hispania Citeriore in eo genere praecipuae. Vid. SCHULTEN 1963 II, 201.

58 Cf. Planchado Portales 1954, 44-45 y 166.

59 CIL II, 3228: municipi Laminitani; CIL II, 3251-52: municipium Flavium Laminitanum. 
tatarse que alcanzaría status municipal ${ }^{60}$ en época ya avanzada, es decir en época flavia. No obstante, de dicha localidad de Alhambra procede una muy interesante pieza epigráfica, ${ }^{61}$ conservada en la actualidad en el Museo local y procedente de la necrópolis de Las Eras donde había sido reutilizada. Se trata de un fragmento de pedestal en honor a Augusto cuyo texto es: [Imp(eratori)] Caesari/Augusto. Divi. f(ilio)/[------]. Pues bien, este pedestal dedicado al primer emperador evidencia la posibilidad de que Laminium se viese potenciado ya desde época augustea, ${ }^{62}$ es decir con anterioridad a la posterior concesión del estatuto de municipium en época flavia.

A pesar de que Ptolomeo (II 6.56) coloca a Laminium entre los núcleos del territorio carpetano, ya Alföldy ${ }^{63}$ propondría que dicha adscripción se trataría de un error del alejandrino, teniendo en cuenta su localización; de esta manera se enmarcaría más bien dentro de los territorios de la Oretania septentrional y no de la Carpetania. ${ }^{64}$ Así pues y a pesar de haber sido situado en Fuenllana por E. Flórez ${ }^{65}$ entre otros, ${ }^{66}$ sin embargo más recientemente se viene localizando en Alhambra, ${ }^{67}$ en el Campo de Montiel, en donde por otro lado se han encontrado numerosos restos arqueológicos ${ }^{68}$ que confirman la entidad de este núcleo.

Otro de los principales ejes en la vertebración del poblamiento de esta área que nos ocupa, y que actuará además como importante eje de comunicación de estos territorios con la alta Andalucía y el Levante, lo vendrá a constituir la vía descrita por los Vasos de Vicarello que ponía en contacto Castulo con Saetabis. ${ }^{69}$ En dicho eje se van a situar dos núcleos destacables en la ordenación territorial de estos ámbitos, como serán Mentesa y Libisosa. En efecto, Mentesa ${ }^{70}$ es citada en el tramo de la vía descrita por los Vasos de Vicarello entre Libisosa ${ }^{71}$ y Mariana de la que le separaban, según

60 McElderry 1918, 74; Galsterer 1971, 42 y 71; Alföldy 1987, 32-37; AbasCAL - EsPinOSA 1989, 74; Mangas 1989, 163; Carrasco Serrano 1999, 309-10; Ortiz de Urbina Álava 2000, 101, nota 194; Domingo Puertas 2001, 151-170; Andreu Pintado 2004, 146, 148 y 153; ID. 2008, 240-242. En relación a la tribu de esta comunidad cf. Wiegels 1985, 152-153; AlföLdy 1987, 35-37. Probablemente y al igual que en otros núcleos de población del interior, la presencia o vinculación con grupos familiares procedentes de otras zonas muy especialmente del levante y en este caso concreto de Saetabis (ALFöLDy 1987, 36-37), vendría a constituir un elemento a tener en cuenta en relación a la propia promoción jurídica de la ciudad, vid., ABASCAL - EsPinOSA 1989, 72; ABASCAL 1996, 277; ID. 2004, 143-145.

61 HEp 17, 2008, nº49; ABASCAL-García BuENo 2013, 296-298.

62 Abascal - García Bueno 2013, 298; también al respecto téngase en cuenta las consideraciones de ANDREu Pintado 2008, 260.

63 AlFÖLDY 1987, 32-33.

64 Esta misma opinión es mantenida por GonzÁlez-Conde1987, 14; RABANAL Alonso - Bragado ToRANZO 1990, 4; por su parte TOVAR 1989, 180, también se refiere a ella junto a los núcleos oretanos, a pesar de afirmar que Ptolomeo la sitúa entre los carpetanos. Véase también al respecto GómEz FraILE 2002, 111 -112.

65 Flórez 1750, V, 22.

66 Vgr. Galsterer 1971, 71.

67 Cf. García y Bellido 1947, 210; Corchado Soriano 1971, 39; Alföldy 1987, 33-34; Tovar 1989, 180 .

68 Véase vgr. Ceán Bermúdez 1832, 42-43; Peñalosa - Martínez Val 1962, 127-130; Corchado SoRIANo 1971, 40-41; Domingo Puertas 2000, 57-61; ID. 2008, 159-182.

69 Vid. Sillières 1977, 31-83; ID. 1990, 261 ss; ID. 1999, 239-250.

70 En cuanto al carácter del nombre, vid., Holder 1904, 549; GARcía Alonso 2003, 351.

71 En dicha fuente (CIL XI, 3281-3284), la distancia que se proporciona entre Mentesa y Libisosa es de XXIIII m.p., en los vasos I, III y IV, mientras que en el II es de XXVIII m.p. 
dicha fuente, XX m.p. Por su parte Ptolomeo (II 6.58) la incluye entre los núcleos de población oretanos. También es citada por Plinio (III 25) entre las comunidades estipendiarias ${ }^{72}$ del conventus Carthaginiensis. De todos modos resulta factible que alcanzase estatuto privilegiado ${ }^{73}$ aunque sea difícil hasta el presente poder establecer con seguridad el momento exacto de su promoción jurídica como municipium. En cuanto a su localización se viene prácticamente de forma unánime situando en Villanueva de la Fuente, ${ }^{74}$ cerca del límite provincial entre Ciudad Real y Albacete. Su territorio de influencia, por tanto, se extendería por el sureste del Campo de Montiel, incluyendo probablemente el área del suroeste de la provincia de Albacete en relación a la vertiente occidental de la Sierra de Alcaraz.

Más hacia el este y en la zona albacetense del Campo de Montiel se situará $L i$ bisosa, que va a constituir el único enclave de rango colonial en estos territorios meridionales de la Meseta. La importante y estratégica situación geográfica de esta colonia augustea determinará, sin duda, que sea mencionada por las principales fuentes antiguas itinerarias, como los Vasos de Vicarello en el eje de comunicación, anteriormente citado ${ }^{75}$ concretamente entre las mansiones de Mentesa y Parietinis. Igualmente es recogida por el Itinerario de Antonino (It. Ant. 446.11: Libisosia) en la vía Item a Laminio alio itinere Caesarea Augusta, distante XIIII m.p., de Caput fluminis Anae, y XXII m.p., de Parietinis, y por el Anónimo de Rávena (313.14) con la forma de Lebinosa. Esta relevante posición geoestratégica del enclave, determinaría que a finales del siglo I a.C., se crease un forum que daría lugar a la colonia Libisosana Foroaugustana ${ }^{76}$ posibilitándose a través de ella un mejor control del tránsito e intenso comercio viario de la zona. ${ }^{77}$ Así pues, Plinio (III 25) cita a Libisosa como colonia cognomine Foroaugustana, ${ }^{78}$ habiendo obtenido según dicho autor

72 Vid. nota 41.

73 Alföldy 1987, 38-39; Abascal - Espinosa 1989, 66, fig. 8; Carrasco Serrano 1999, 320; Andreu Pintado 2004, 146, 148 y 154; ID. 2008, 251-252. Véase también comentario de ABASCAL 1990, 29, a la inscripción procedente del término de Alcaraz; en el mismo sentido también vid. Noguera CELDRÁN 1992, 38-39. Por otra parte el hecho de que Mentesa llegara a ser en época tardía sede episcopal, reafirmaría según AlFöldy (1987, 39), haber llegado a alcanzar con anterioridad status de núcleo privilegiado.

74 Véase vgr. SAavedra 1862, 20; Hervás y Buendía 1899, II, 609-610; Miller 1916, 181; García Y Bellido 1947, 220; Roldán Hervás 1975, 250; Alföldy 1987, 41; Sillières 1990, 273. Por otra parte y en relación a los numerosos hallazgos de materiales arqueológicos encontrados en dicha localidad de Villanueva de la Fuente, véanse las diversas aportaciones recogidas en BENíteZ DE LUGO 2001.

75 Vid. nota 69.

76 Según BlÁzQUEz (1991, 202), algunos fora darían lugar a la fundación de colonias, como sería el caso del Forum Augustanorum que se convirtió en la colonia Libisosana Foroaugustana mencionada por Plinio (III 25). Más recientemente y en este mismo sentido véase Uroz SÁEz 2012, 103-105. También aun cuando matizándose la propuesta tradicional cronológica para la fundación de la colonia, cf. PovEDA NAVARRo 2002, 5-38.

77 En relación a la organización viaria en el territorio del entorno a Libisosa vid. CoRzo SÁNCHEz 1976, 228-229.

78 Se viene considerando tradicionalmente como fundación colonial augustea, vid. ViTTINGHOFF 1952 , 107; García y Bellido 1959, 494-5; Galsterer 1971, 71; Blázquez 1976, 116; Alföldy 1987, 31-32; Solana Sáinz 1989, 85; Abascal-Espinosa 1989, 65; Olivares Pedreño 1998, 217. No obstante y para una propuesta sobre la promoción a colonia de Libisosa en un momento posterior a Augusto pero anterior a los Flavios, véase Poveda Navarro 2002, 24-32. Sin embargo más recientemente vid., las consideraciones al respecto de ANDreu PinTAdo 2008, 222-233, nota 35. También sobre esta problemática véase Uroz SÁEZ 2012, 105-109. 
clásico derecho itálico ${ }^{79}$ Está asimismo atestiguada epigráficamente como colonia Libisosanorum,$^{80}$ pudiéndose constatar también a través de la epigrafía ${ }^{81}$ la pertenencia de los ciudadanos a la tribu Galeria. ${ }^{82}$ Por su parte Ptolomeo en el libro II de su obra geográfica ${ }^{83}$ la recoge entre los núcleos de población oretanos. Por otro lado, resulta prácticamente unánime su localización en torno a Lezuza (Cerro del Castillo) desde Flórez, ${ }^{84}$ Cortés y López, ${ }^{85}$ Saavedra ${ }^{86}$ y Miller ${ }^{87}$ entre otros, y posteriormente por García y Bellido, ${ }^{88}$ para quien el nombre actual sería corrupción del antiguo, ${ }^{89}$ Roldán Hervás, ${ }^{90}$ Alföldy, ${ }^{91}$ A. Tovar ${ }^{92}$ y P. Sillières. ${ }^{93}$ Por su parte las sucesivas campañas arqueológicas que desde 1996 se vienen llevando a cabo en el "Cerro del Castillo" en Lezuza, están permitiendo poner de manifiesto la importancia de este enclave. Tanto, pues, la documentación arqueológica ${ }^{94}$ procedente de Libisosa, como su estratégica posición, nos posibilitan reconocer su destacable papel en la ordenación territorial, desempeñando funciones de centro de un amplio y extenso territorium en el ámbito occidental de la provincia de Albacete. ${ }^{95}$

A partir, por tanto, del siglo I d.C., el programa de actuación de Augusto se dejaría sentir en estas tierras de la Submeseta sur a través de la consolidación, promoción e integración jurídica de toda una serie de núcleos de población. Núcleos estos que se van a situar en torno a una serie de ejes de comunicación, fundamentales en la articulación y vertebración del poblamiento, en unos ámbitos territoriales como estos de no muy amplia densidad poblacional, pero sí de gran importancia estratégica como zona de paso obligado entre el Mediodía, la zona del Levante y el interior peninsular.

\footnotetext{
79 Plin., III 25: Carthaginem conveniunt populi LXV exceptis insularum incolis: ex colonia Accitana Gemellenses, ex Libisosana cognomine Foroaugustana quibus duabus ius Italiae datum.

80 CIL II, 3234. ViVES 1971, 132, nº1133; AbASCAL 1990, 43-44.

81 CIL II, 4254. VIVES 1971, 188, nº1617; AlFÖLDY 1973, 91, nº70; ID. 1975, 172, n³13; también véase ETIENNE 1958, 132 y 139.

82 Vid. KubitscheK 1882, 175-176; Wiegels 1985, 120.

83 Ptol., II 6.58.

84 FLóREZ 1769, XXIV, 177.

85 CORTÉs y LÓPEZ 1836, 134-135.

86 SAAVEDRA 1862, 97.

87 Miller 1916, 181.

88 GARCÍA y BELLIDO 1947, 238; ID. 1959, 494-495.

89 También y en relación al nombre véase Holder 1904, 205; GARCía AlOnSO 2003, 248-249.

90 RoldÁn Hervás 1975, 246.

91 Alföldy 1987, 31-32.

92 TOVAR $1989,178$.

93 Sillières 1977, 63 y 75; ID. 1990, 273.

94 Vid. Uroz Sáez et alI 2002, 245-251; Uroz SÁez - Márquez Villora 2002, 239-244; Muñoz OjeDA 2004, 553-557; Uroz SÁEz ET ALII 2004, 181-191; Uroz SÁEZ ET ALII 2006, 173-184; PovedA NaVARRo ET aLII 2008, 481-497; Hernández Canchado 2008, 143-177; Uroz SÁez 2012, 87-130.

95 Por el suroeste limitaría con el territorio de Mentesa, y por el oeste con el territorio de Laminium.
} 


\section{Bibliografía}

Abad Casal, L. - Bendala Galán, M. (1997): "Urbanismo y ciudad: de las formaciones ibéricas a la consolidación del modelo romano", [en] Actas del XXIII Congreso Nacional de Arqueología, II, Elche, 11-20.

ABASCAL, J. M.

(1990): Inscripciones romanas de la provincia de Albacete, Albacete.

(1996): "Derecho latino y municipalización en Levante y Cataluña", [en] E. Ortiz de Urbina - J. Santos (eds.), Teoría y práctica del ordenamiento municipal en Hispania, Vitoria, 255-283.

(2004): "Élites y sociedad romana de la Meseta sur", [en] Epigrafia y sociedad en Hispania durante el Alto Imperio: estructuras y relaciones sociales, Alcalá de Henares, 141-158.

(2006): "Los tres viajes de Augusto a Hispania y su relación con la promoción jurídica de ciudades", Iberia 9, 63-78.

Abascal, J. M. - EsPINosa, U. (1989): La ciudad hispano-romana: privilegio y poder, Logroño.

Abascal, J. M. - García Bueno, C. (2013): “Inscripciones de Agudo (Baetica, Conventus Cordubensis) y Alhambra (Hispania Citerior, Conventus Carthaginiensis)", ZPE 184, 293 298.

Albertini, E. (1923): Les divisions administratives de l'Espagne romaine, Paris.

ALFÖLDY, G.

(1973): Flamines provinciae Hispaniae Citerioris, Madrid.

(1975): Die römischen Inschriften von Tarraco, Berlín.

(1987): Römisches Städtewesen auf der neukastilischen Hochebene. Ein Testfall für die Romanisierung, Heidelberg.

(1987a): "Epigraphica Hispanica IX. Inschriften aus Ciudad Real”, ZPE 67, 225-248.

(1999): “Aspectos de la vida urbana en las ciudades de la Meseta sur", [en] J. González (ed.), Ciudades privilegiadas en el Occidente romano, Sevilla, 467-485.

ANDREU PinTado, J.

(2004): Edictum, municipium y lex: Hispania en época Flavia (69-96 d.C.), Oxford.

(2008): "Municipalización y vida municipal en las comunidades romanas de la Meseta sur", [en] G. Carrasco Serrano (coord.), 225-260.

Baratta, G. (2001): Il culto di Mercurio nella Penisola Iberica, Barcelona.

Bendala Galán, M.

(1990): "El plan urbanístico de Augusto en Hispania: precedentes y pautas macroterritoriales", [en] Stadtbild und Ideologie. Die Monumentalisierung hispanischer Städte zwischen Republik und Kaiserzeit, München, 25-42.

(1998): "Fórmulas de promoción y desarrollo urbano y urbanístico en la Hispania tardorrepublicana", [en] J. Mangas (ed.), Italia e Hispania en la crisis de la República romana, Madrid, 307-312.

Benítez de Lugo, L. (dir.) (2001): Mentesa Oretana 1998-2000, Ciudad Real.

BLÁZQUEZ, A.

(1892): "Nuevo estudio sobre el Itinerario de Antonino", BRAH 21, 51-125.

(1912): "Vías romanas de la Beturia de los Túrdulos", BRAH 61, 359-370. 
BlÁZQUeZ, J. M ${ }^{\mathrm{a}}$

(1976): "Ciudades hispanas de la época de Augusto", [en] Symposion de ciudades augusteas, I, Zaragoza, 79-136.

(1991): "El urbanismo en Occidente”, [en] J. Ma Blázquez (ed.), Urbanismo y Sociedad en Hispania, Madrid, 205-221.

Canto, A. M. (1993): “De situ Siarensium Fortunalium: corrección a Plinio, N.H. III, 13-14 (Baeturia Celticorum)", CuPAUAM 20, 171-184.

Carrasco Serrano, G.

(1990): "Introducción al estudio de las vías romanas de la provincia de Ciudad Real", [en] Simposio sobre la red viaria en la Hispania romana, Zaragoza, 85-93.

(1997): "Sobre CIL, II, 3270 y la antigua vía romana de comunicación Castulo-Sisapo", [en] Actas III Congreso Internacional de Caminería Hispánica, Madrid, 183-191.

(1999): "Sobre los municipia del ámbito territorial castellano-manchego", ETF (II) 12, 309-323.

(2007): "Vías de comunicación y moneda en torno a Sisapo en época romana", [en] Necedad, sabiduría y verdad: el legado de Juan Cascajero, (=Gerión extra 1), 363-373.

(2008): "La intervención romana e n Castilla-La Mancha: la anexión del territorio", [en] Carrasco Serrano, G. (coord.), 13-32.

(2011): "Vías y mansiones romanas en el territorio del Campo de Montiel", HAnt. 35, 321325.

Carrasco Serrano, G. (coord.)

(2008): La romanización en el territorio de Castilla-La Mancha, Cuenca. (2012): La ciudad romana en Castilla-La Mancha, Cuenca.

CeÁn Bermúdez, J. A. (1832): Sumario de las antigüedades romanas que hay en España, Madrid.

Chic GArcía, G. (1991): “Estrabón y la práctica de la amalgama en el marco de la minería sudhispánica: un texto mal interpretado", [en] La Bética en su problemática histórica, Granada, 7-29.

Coello, F. (1889): "Vías romanas entre Toledo y Mérida", BRAH 15, 5-42.

Contreras de la Paz, R. (1965): “Un gran bienhechor de Castulo: Quinto Torio Culeón”, Oretania 20, 63-96.

Corchado Soriano, M.

(1969a): El camino de Toledo a Córdoba, Jaén.

(1969b): "Estudio sobre vías romanas entre el Tajo y el Guadalquivir", AEspA 42, 124-158.

(1971): Avance de un estudio geográfico-histórico del Campo de Montiel, Madrid.

(1982): Estudio del Campo de Calatrava, III. Los pueblos y sus términos, Ciudad Real.

Corell, J. (1996): "Dedicatoria a Mercurio hallada en Alhambra (Ciudad Real)", Ficheiro Epigráfico 52, 3-5.

CoRTÉS y LóPEz, M. (1836): Diccionario geográfico-histórico de la España Antigua, III, Madrid.

Corzo Sánchez, R. (1976): "In finibus emeritensium”, [en] Actas del Simposio del Bimilenario de Mérida "Augusta Emerita”, Madrid, 217-233.

Corzo SÁnchez, R. - Toscanos SAn GiL, M. (1992): Las vías romanas de Andalucía, Sevilla.

CURChIN, L.A. (1997): “Celtization and romanization of toponymy in central Spain”, Emerita $65,257-279$. 
Domergue, C.

(1990): Les mines de la Péninsule Ibérique dans l'antiquité romaine, Roma.

(2008): Les mines antiques; les productions des métaux aux époques grecque et romaine, Paris.

Domingo Puertas, L. A.

(2000): "En torno al problema de la localización de Laminium: últimas aportaciones", HAnt. 24, 45-63.

(2001): "La ciudad iberromana de Laminium: evolución y municipalización", HAnt. 25, 151-170.

(2008): "Fuentes epigráficas para el estudio del municipio iberromano de Laminium (Alhambra, Ciudad Real)", HAnt. 32, 159-182.

Duncan-Jones, R. P. (1974): “The Procurator as Civic Benefactor”, JRS 64, 79-85.

Etienne, R. (1958): Le culte imperial dans la Péninsule Ibérique d'Auguste a Dioclétien, Paris.

FERNÁNDEZ OCHOA, C. ET ALII

(1982-1983): "Nuevo documento epigráfico para la localización de Sisapo", CuPAUAM 9-10, 211-220.

(1994): Sisapo I. Excavaciones arqueológicas en "La Bienvenida”, Almodóvar del Campo (Ciudad Real), Toledo.

(2002): Arqueominería del sector central de Sierra Morena. Introducción al estudio del Área Sisaponense, Madrid.

FITA, F. (1910): “Lápida romana de Almadén”, BRAH 56, 527-528.

FLórez, E. (1747-1775): España Sagrada, Madrid.

GALSTERER, H. (1971): Untersuchungen zum römischen Städtewesen auf der Iberischen Halbinsel, Berlín.

GARCÉs, A. M ET ALII (2000): "Yacimiento arqueológico de Nuestra Señora de Oreto-Zuqueca", [en] El Patrimonio arqueológico de Ciudad Real, Valdepeñas, 241-255.

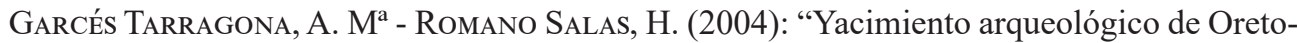
Zuqueca", [en] Investigaciones Arqueológicas en Castilla-La Mancha 1996-2002, Toledo, 307-324.

García Alonso, J. L. (2003): La Península Ibérica en la Geografía de Claudio Ptolomeo, Vitoria.

(2008): "Romanización y celtización en la toponimia de la Meseta sur", [en] G. Carrasco Serrano (coord.), 339-366.

García Iglesias, L. (1971): "La Beturia, un problema geográfico de la Hispania Antigua", AEspA 44, 86-108.

García y Bellido, A.

(1945): España y los españoles hace dos mil años según la Geografía de Estrabón, Madrid.

(1947): La España del siglo primero de nuestra Era, Madrid.

(1953): La Península Ibérica en los comienzos de la historia, Madrid.

(1959): "Las colonias romanas de Hispania", AHDE 29, 447-512.

Gimeno Pascual, H. (2008): "Paisajes epigráficos en el espacio romano de la Comunidad de Castilla-La Mancha”, [en] G. Carrasco Serrano (Coord.), 2008, 261-338. 
Gómez FraILE, J. Ma (2002): "Elementos para la definición del espacio geográfico de los carpetanos", Revista de la C.E.C.E.L. 2, 93-118.

González-Conde, Ma P. (1987): Romanidad e indigenismo en Carpetania, Alicante.

González Román, C. - Mangas, J. (1991): Corpus de Inscripciones latinas de Andalucía, III, Jaén.

HernáNDez CANCHADO, N. (2008): “La cerámica de importación tardorrepublicana del barrio iberorromano de Libisosa: el Departamento 79", Verdolay 2, 143-177.

Hervás y BuENDÍA, I. (1899): Diccionario histórico, geográfico, biográfico y bibliográfico de la provincia de Ciudad Real, $2^{\mathrm{a}}$ ed., Madrid.

Holder, A. (1904): Alt-Celtischer Sprachschatz, II, Leipzig.

Humphrey, J. H. (1986): Roman Circuses. Arenas for Chariot Racing, London.

KuBITSCHEK, W. (1882): De romanorum tribuum origine ac propagatione, Wien.

Madoz, P. (1846): Diccionario geográfico-estadístico-histórico de España y sus posesiones de Ultramar, T. II, Madrid.

Marín Díaz, N. - Prieto Arciniega, A. (1974): "En torno a un nuevo planteamiento de los límites de la provincia romana de la Bética", HAnt. 4, 77-86.

MCElderry, R. K. (1918): “Vespasian's reconstruction of Spain”, JRS 8, 53-102.

Melchor Gil, E.

(1993): "Vías romanas y explotación de los recursos mineros de la zona norte del Conventus Cordubensis", Anales de Arqueología Cordobesa 4, 63-89.

(1995): Vías romanas de la provincia de Córdoba, Córdoba.

(1999): "Élites municipales y mecenazgo cívico en la Hispania romana", [en] F. J. Rodríguez Neila - F. J. Navarro (eds.), Élites y promoción social en la Hispania romana, Pamplona, 219-263.

Miller, K. (1916): Itineraria Romana. Römische Reisewege an der Hand Tabula Peutingeriana, Stuttgart.

Muñoz OJedA, J. (2004): "Programa decorativo de un edificio singular en el foro de Libisosa”, [en] S. F. Ramalló (ed.), La decoración arquitectónica en las ciudades romanas de Occidente, Murcia, 553-557.

Nieto Gallo, G. et aliI (1980): Oreto I, Madrid.

Noguera Celdrán, J. M. (1992): "Algunas consideraciones sobre tres nuevos documentos del arte sepulcral romano-provincial popular en Albacete", Al-Basit 31, 19-46.

Olivares Pedreño, J. C. (1998): Conflicto político y promoción jurídica de comunidades en el Occidente romano (133 a.C.-74 d.C.), Alicante.

Ortiz de Urbina Álava, E. (2000): Las comunidades hispanas y el derecho latino. Observaciones sobre los procesos de integración local en la práctica político-administrativa al modo romano, Vitoria.

OzCÁRIz GIL, P. (2013): La administración de la provincia Hispania Citerior durante el Alto Imperio romano, Barcelona.

Peñalosa, M. - Martínez VAl, J. Ma (1962): "Hallazgos arqueológicos en Alhambra”, Cuadernos de Estudios Manchegos 12, 127-130.

Piernavieja, P. (1977): Corpus de inscripciones deportivas de la España romana, Madrid.

PlácIDo, D. (2008): Las provincias hispanas durante el Alto Imperio romano, Madrid. 
Planchuelo Portales, G. (1954): Estudio del alto Guadiana y de la Altiplanicie del Campo de Montiel, Madrid.

Poveda Navarro, A. M. (2002): "Fora Hispana: la evidencia de Libisosa Forum Augustum (Lezuza, Albacete)", Conimbriga 41, 5-38.

Poveda Navarro, A. M. et aliI (2008): "Hallazgos escultóricos en la colonia romana de $L i$ bisosa (Lezuza, Albacete)", [en] J. M. Noguera - E. Conde (eds.), Actas V Reunión sobre escultura romana en Hispania, Murcia, 481-497.

Prieto Arciniega, A. (1973): Estructura social del Conventus Cordubensis durante el Alto Imperio Romano, Granada.

Rabanal Alonso, M. A. - Bragado Toranzo, J. Ma (1990): "Fuentes antiguas sobre Carpetania”, [en] Toledo y la Carpetania en la Edad Antigua, Toledo, 19-37.

RoLdÁn HERvás, J.M.

(1975): Itineraria Hispana. Fuentes antiguas para el estudio de las vías romanas en la Península Ibérica, Valladolid-Granada.

(1982): "La organización político-administrativa y judicial de la Hispania romana", [en] $H^{a}$ de España de R. Menéndez Pidal, II/2, Madrid, 83-131.

SaAvedra, E. (1862): Discursos leídos ante la Real Academia de la Historia, Madrid.

SCHULTEN, A.

(1952): Fontes Hispaniae Antiquae VI. Estrabón. Geografia de Iberia, Barcelona.

(1963): Geografía y etnografía antiguas de la Península Ibérica, II, Madrid.

SILLIÈRES, P.

(1977): "Le Camino de Aníbal. Itinéraire des gobelets de Vicarello, de Castulo à Saetabis", MCV 13, 31-83.

(1980): "Sisapo: prospections et decouvertes", AEspA 53, 49-57.

(1990): Les voies de communication de l'Hispanie méridionale, Paris.

(1999): "Le Camino de Aníbal, principal axe des communications entre l'est et le sud de Hispanie", [en] R. M. S. Centeno et alii (coords.), Rutas, ciudades y moneda en Hispania, Madrid, 239-250.

Solana SÁrnz, J. Ma (1989): “Colonización y municipalización bajo César y Augusto: Hispania Citerior", [en] Aspectos de la colonización y municipalización de Hispania, Mérida, 71-106.

STYLOW, A. U.

(1985): “Ordenación territorial romana en el valle de los Pedroches (Conventus Cordubensis)”, [en] Actas XVII Congreso Nacional de Arqueología, Zaragoza, 657-666.

(1991): "El municipium flavium V(---) de Azuaga (Badajoz) y la municipalización de la Baeturia Turdulorum", SHHA 9, 11-27.

(2000): "Die Accitani veteres und die Kolonie Iulia Gemella Acci. Zum Problem von veteres, Alt-Stadt und Kolonie in der Hispania Ulterior", Chiron 30, 775-806.

Thouvenot, R. (1940): Essai sur la province romaine de Bétique, Paris.

Tovar, A. (1989): Iberische Landeskunde. Las tribus y las ciudades de la antigua Hispania. 3. Tarraconensis, Baden-Baden.

Uroz SÁEz, J. (2012): "La colonia romana de Libisosa y sus precedentes", [en] G. Carrasco Serrano (coord.), 87-130. 
UROZ SÁEZ, J. ET ALII

(2002): "El foro de Libisosa. Datos preliminares de una investigación en curso", [en] Actas II Congreso de Historia de Albacete, I, Albacete, 245-251.

(2004): “Aproximación al conjunto arqueológico y monumental de Libisosa (Cerro del Castillo, Lezuza, Albacete)", [en] Investigaciones Arqueológicas en Castilla-La Mancha, 1996-2002, Toledo, 181-191.

(2006): "La Puerta Norte de Libisosa: cronología y arquitectura", [en] Th. Schattner - F. Valdés (eds.), Stadttore Bautyp und Kunstform. Puertas de ciudad. Tipo arquitectónico y forma artística, Toledo, 173-184.

Uroz SÁEz, J. - MÁrquez Villora, J.C. (2002): "La puerta norte de Libisosa y su contexto arqueológico", [en] Actas II Congreso de Historia de Albacete, I, Albacete, 239-244.

VelazA, J. (2012): "Epigrafía y autorrepresentación cívica en las ciudades romanas de la Meseta sur: algunos apuntes”, [en] G. Carrasco Serrano (coord.), 397-413.

Ventura Villanueva, A. (1993): "Susum ad Montes S(ocietatis) S(isaponensis): nueva inscripción tardorrepublicana de Córdoba", Anales de Arqueología Cordobesa 4, 49-61.

VILlAR, F. (2000): Indoeuropeos y no indoeuropeos en la Hispania prerromana, Salamanca.

VitTinghoff, F. (1952): Römische Kolonisation und Bürgerrechtspolitik unter Caesar und Augustus, Wiesbaden.

ViVES, J. (1971): Inscripciones latinas de la España romana, Barcelona.

WiEgels, R. (1985): Die Tribusinschriften des römischen Hispanien, Berlín.

Zarzalejos Prieto, M. et aLII

(1994): "Excavaciones en La Bienvenida (Ciudad Real). Hacia una definición preliminar del horizonte histórico-arqueológico de la Sisapo antigua", [en] Arqueología en Ciudad Real. Jornadas de Arqueología de Ciudad Real en la UAM, Toledo, 167-194.

(2004): "El proyecto Sisapo-La Bienvenida (Almodóvar del Campo, Ciudad Real). Balance de los trabajos más recientes y nuevas perspectivas de la investigación", [en] Investigaciones Arqueológicas en Castilla-La Mancha 1996-2002, Toledo, 163-180. 